\title{
Gödel universe from string theory
}

\author{
Shou-Long Li ${ }^{1, \mathrm{a}}$, Xing-Hui Feng ${ }^{2, \mathrm{~b}}$, Hao Wei ${ }^{1, \mathrm{c}}, \mathrm{H}$. Lï $^{2, \mathrm{~d}}$ \\ ${ }^{1}$ School of Physics, Beijing Institute of Technology, Beijing 100081, China \\ 2 Department of Physics, Center for Advanced Quantum Studies, Beijing Normal University, Beijing 100875, China
}

Received: 22 February 2017 / Accepted: 24 April 2017 / Published online: 6 May 2017

(C) The Author(s) 2017. This article is an open access publication

\begin{abstract}
The Gödel universe is a direct product of a line and a three-dimensional spacetime we call $\mathrm{G}_{\alpha}$. In this paper, we show that the Gödel metrics can arise as exact solutions in Einstein-Maxwell-Axion, Einstein-Proca-Axion, or Freedman-Schwarz gauged supergravity theories. The last option allows us to embed the Gödel universe in string theory. The ten-dimensional spacetime is a direct product of a line and the nine-dimensional one of an $S^{3} \times S^{3}$ bundle over $\mathrm{G}_{\alpha}$, and it can be interpreted as some decoupling limit of the rotating D1/D5/D5 intersection. For some appropriate parameter choice, the nine-dimensional metric becomes an $\mathrm{AdS}_{3} \times S^{3}$ bundle over squashed 3-sphere. We also study the properties of the Gödel black holes that are constructed from the double Wick rotations of the Gödel metrics.
\end{abstract}

\section{Contents}

1 Introduction . . . . . . . . . . . 1

2 Gödel universe . . . . . . . . . . . . . . . . 2

2.1 The metrics of $\mathrm{G}_{\alpha} \times \mathbb{R} \ldots \ldots \ldots 2$

2.2 Energy condition and $\alpha$ value . . . . . . . . 2

2.3 Metrics asymptotic to $\mathrm{G}_{\alpha} \ldots \ldots \ldots . \ldots 3$

2.4 Mass and angular momentum . . . . . . . . . 3

2.5 Squashed 3-sphere $S_{\alpha}^{3} \times \mathbb{T} \ldots \ldots . \ldots 3$

3 Gödel solutions from Lagrangian formalism . . . . 4

3.1 Einstein-Maxwell-Axion theory with a topological term . . . . . . . . . . . 4

3.2 Einstein-Proca-Axion theory . . . . . . . . . 4

3.3 The embedding of squashed 3-sphere . . . . . 5

3.4 Generalizing to higher dimensions . . . . . . 5

3.5 Effective three-dimensional theories . . . . . 5

4 Black holes from double Wick rotations . . . . . . 6

\footnotetext{
a e-mail: sllee_phys@bit.edu.cn

be-mail: xhfengp@mail.bnu.edu.cn

ce-mail: haowei@bit.edu.cn

de-mail: mrhonglu@gmail.com
}

4.1 Type I . . . . . . . . . . . . . . . 6

4.2 Type II . . . . . . . . . . . . . . . . . . . . 6

5 Embedding in string theory $\ldots \ldots \ldots 7$

5.1 Freedman-Schwarz model . . . . . . . . . . 7

5.2 Gödel universe from string theory $\ldots \ldots .7$

$5.3 \mathrm{AdS}_{3} \times S^{3}$ bundle over $S_{\alpha}^{3} \ldots \ldots \ldots 8$

6 Conclusions ............... 8

References ................... 9

\section{Introduction}

Closed time-like curves (CTCs) in solutions of General Relativity can pose challenges to Hawking's chronology protection conjecture. It is well known that the Kerr metric has CTCs; however, they are hidden inside the event horizon, and hence they obey the chronological censorship. The Gödel metric [1], a solution of cosmological Einstein gravity coupled to some uniform pressureless matter, describes a homogeneous rotating universe that has naked CTCs and has no globally spatial-like Cauchy surface. However, the finetuning balance between the cosmological constant and the matter density in the Gödel universe makes it an unrealistic model to challenge the chronology protection. Nevertheless it is a good toy model to study the effects of naked CTCs in both classical and quantum gravities.

The study of CTCs has enjoyed considerable attention with the development of the string theory and the AdS/CFT correspondence. A large number of supersymmetric or nonsupersymmetric Gödel-like solutions, including also black holes and time machines, with naked CTCs were constructed in gauged supergravities in higher dimensions, see e.g. [217]. This work raises important issues of whether the problems of CTCs may be resolved by stringy or quantum considerations, or whether naked CTCs in the bulk implt the breakdown of unitarity of the field theory.

In this paper, we focus on the original four-dimensional Gödel universe, which is a direct product of a line and a three-dimensional metric [1], 
$\mathrm{d} s^{2}=\ell^{2}\left(-(\mathrm{d} t+r \mathrm{~d} \phi)^{2}+\frac{1}{2} r^{2} \mathrm{~d} \phi^{2}+\frac{\mathrm{d} r^{2}}{r^{2}}+\mathrm{d} z^{2}\right)$.

This metric is an exact solution of the Einstein equation involving some uniform pressureless matter,

$$
\begin{array}{r}
R_{\mu \nu}-\frac{1}{2} R g_{\mu \nu}+\Lambda g_{\mu \nu}=T_{\mu \nu}^{\mathrm{mat}}, \\
\Lambda=-\frac{1}{2 \ell^{2}}, \quad T_{\mu \nu}^{\mathrm{mat}}=u_{\mu} u_{\nu},
\end{array}
$$

where $u^{\mu}=\left(\ell^{-2}, 0,0,0\right)$. The Gödel universe has aroused continuous interest in the general-relativity community, (see, e.g., [18-24]), and the Gödel metric (1.1) was generalized to Gödel-type metrics where the coefficient $\frac{1}{2}$ in (1.1) is replaced by a generic constant $\alpha$. To avoid pedantry, we shall refer to all these metrics as Gödel metrics, which are distinct from the afore-mentioned Gödel-like metrics. It turns out that most theories associated with these general Gödel metrics involve the energy-momentum tensor of some uniform pressureless matter. Electric and magnetic fields periodic in $z$ as a replacement for such matter were used to construct Gödel metric in [21]. It is worth mentioning that the threedimensional metric with $\mathrm{d} z^{2}$ removed can arise as an exact solution of Einstein-Maxwell theory with a topological term $A \wedge F$ [13]. The embedding of the three-dimensional metric in heterotic string theory was first given in [11].

The main purpose of this paper is to construct more Lagrangians that admit Gödel metrics as exact solutions. We present three Lagrangians that admit the Gödel metrics as solutions, all involving only the fundamental matter fields. These are the Einstein-Maxwell-Axion (EMA), EinsteinProca-Axion (EPA) and Freedman-Schwarz [25] $S U(2) \times$ $S U$ (2) gauged supergravity theories. The Freedman-Schwarz model can be obtained from the effective actions of string theories via the Kaluza-Klein reduction on $S^{3} \times S^{3}$. Thus the four-dimensional Gödel universe can be embedded in string theory.

The paper is organized as follows. In Sect. 2, we give a review of Gödel metrics and their properties. We also show that for an appropriate choice of the parameter, such a metric can also describe a direct product of time and a squashed 3sphere. In Sect. 3, we construct EMA and EPA theories that admit Gödel metrics, and also solutions involving a squashed 3 -sphere. We generalize the theories to higher dimensions and also give the effective three-dimensional theories by Kaluza-Klein reduction. In Sect. 4, we consider double Wick rotations and obtain two types of black hole solutions. In Sect. 5, we show that Gödel metrics are exact solutions of the Freedman-Schwarz model and hence obtain the corresponding ten-dimensional solutions of string theories. We conclude the paper in Sect. 6.

\section{Gödel universe}

\subsection{The metrics of $\mathrm{G}_{\alpha} \times \mathbb{R}$}

In this paper, we consider a class of metrics in the following form:

$$
\mathrm{d} s^{2}=\ell^{2}\left(-(\mathrm{d} t+r \mathrm{~d} \phi)^{2}+\alpha r^{2} \mathrm{~d} \phi^{2}+\frac{\mathrm{d} r^{2}}{r^{2}}+\mathrm{d} z^{2}\right),
$$

where $\ell$ and $\alpha$ are constants. The metric is a direct product of $\mathbb{R}$ associated with the coordinate $z$ and the three-dimensional metric of $(t, \phi, r)$, which we shall call $\mathrm{G}_{\alpha}$. The original Gödel metric [1] is recovered when we take $\alpha=\frac{1}{2}$, corresponding to $G_{1 / 2} \times \mathbb{R}$. To avoid pedantry, we shall refer the metric (2.1) with generic $\alpha$ also as the Gödel metric.

In addition to the constant shifting symmetry along the $(t, \phi, z)$ directions, the Gödel metric (2.1) is invariant under the constant scaling

$r \rightarrow \lambda r, \quad \phi \rightarrow \lambda^{-1} \phi$.

Note that imposing this scaling symmetry also implies that $\phi$ describes a real line, rather than a circle. This scaling property indicates that the metric is homogeneous. Since the metric $\mathrm{G}_{\alpha}$ is three dimensional, its curvature is completely determined by the Ricci tensor, whose non-vanishing components are given by

$R^{\overline{0} \overline{0}}=\frac{1}{2 \alpha \ell^{2}}, \quad R^{\overline{1} \overline{1}}=\frac{1-2 \alpha}{2 \alpha \ell^{2}}=R^{\overline{2} \overline{2}}$.

Here we represent the curvature in tangent space by the vielbein

$e^{\overline{0}}=\ell(\mathrm{d} t+r \mathrm{~d} \phi), \quad e^{\overline{1}}=\ell \sqrt{\alpha} r \mathrm{~d} \phi, \quad e^{\overline{2}}=\frac{\ell \mathrm{d} r}{r}$,

$e^{\overline{3}}=\ell \mathrm{d} z$.

It is clear that when $\alpha=1$, the metric is locally $\mathrm{AdS}_{3}$ (threedimensional anti-de Sitter spacetime), i.e. $\mathrm{G}_{1}=\mathrm{AdS}_{3}$.

\subsection{Energy condition and $\alpha$ value}

Defining the energy-momentum tensor in the vielbein basis,

$T^{a b}=R^{a b}-\frac{1}{2} \eta^{a b} R$,

we find $T^{a b}=\operatorname{diag}\{\rho, p, p, \tilde{p}\}$, with

$\rho=\frac{3-4 \alpha}{4 \alpha \ell^{2}}, \quad p=\frac{1}{4 \alpha \ell^{2}}, \quad \tilde{p}=\frac{4 \alpha-1}{4 \alpha \ell^{2}}$.

Thus one sees that $\alpha=\frac{1}{2}$ gives rise to matter with uniform pressure [1]. The null-energy condition requires that

$\rho+p=\frac{1-\alpha}{\alpha \ell^{2}} \geq 0, \quad \rho+\tilde{p}=\frac{1}{2 \alpha \ell^{2}} \geq 0$. 
Thus the null-energy condition requires that $0<\alpha \leq 1$. As we shall see presently this implies that Gödel metrics in general have naked CTCs.

\subsection{Metrics asymptotic to $\mathrm{G}_{\alpha}$}

We now consider deformations of $\mathrm{G}_{\alpha}$ by introducing a function of two constants,

$f=1+\frac{a}{r}-\frac{b}{r^{2}}$,

and deform the metric (2.1) to become

$\mathrm{d} s^{2}=\ell^{2}\left(-(\mathrm{d} t+r \mathrm{~d} \phi)^{2}+\alpha r^{2} f \mathrm{~d} \phi^{2}+\frac{\mathrm{d} r^{2}}{r^{2} f}+\mathrm{d} z^{2}\right)$.

It is straightforward to verify that the curvature tensors (2.3) remain unchanged. This implies that the metric (2.9) is locally the same as (2.3). However, globally, the deformed metric (2.9) is different from (2.1). An important difference is that in the deformed metric (2.9), the coordinate $\phi$ is periodic, namely

$\Delta \phi=\frac{4 \pi}{\sqrt{\alpha} r_{0}^{2} f^{\prime}\left(r_{0}\right)}$, with $f\left(r_{0}\right)=0$.

This ensures that the metric is absent from a conical singularity at $r=r_{0}$. After imposing this condition, the coordinate transformation that relates (2.1) to (2.9) breaks the scaling symmetry (2.2) and hence the two metrics are not equivalent globally.

To demonstrate this explicitly, we note that the parameter $a$ is trivial in that it can be eliminated by the coordinate transformation $r \rightarrow r-\frac{1}{2} a$ and $t \rightarrow t+\frac{1}{2} a \phi$, without altering the global structure. The positive parameter $b$ can be set to 1 without loss of generality, using the scaling $r \rightarrow \sqrt{b} r$, together with appropriate scalings of the rest coordinates. Now let $r=\cosh \hat{r}, \phi=\hat{\phi} / \sqrt{\alpha}, t=\hat{t}-\hat{\phi} / \sqrt{\alpha}$, the metric (2.9) with $a=0$ and $b=1$ becomes

$$
\begin{aligned}
\mathrm{d} s^{2}= & \ell^{2}\left(-\left(\mathrm{d} \hat{t}+\frac{2}{\sqrt{\alpha}} \sinh ^{2}\left(\frac{1}{2} \hat{r}\right) \mathrm{d} \hat{\phi}\right)^{2}\right. \\
& \left.+\sinh ^{2} \hat{r} \mathrm{~d} \hat{\phi}^{2}+\mathrm{d} \hat{r}^{2}+\mathrm{d} z^{2}\right) .
\end{aligned}
$$

On the other hand, we find that, under the coordinate transformation

$r=\cosh \hat{r}+\cos \hat{\phi} \sinh \hat{r}, \quad r \phi=\frac{1}{\sqrt{\alpha}} \sin \hat{\phi} \sinh \hat{r}$,

$\tan \left(\frac{1}{2} \hat{\phi}+\frac{1}{2} \sqrt{\alpha}(t-\hat{t})\right)=\frac{1}{\hat{r}} \tanh \left(\frac{1}{2} \hat{\phi}\right)$,

the metric (2.1) becomes also precisely (2.11). (This coordinate transformation reduces to the one obtained in [1] for $\alpha=\frac{1}{2}$.) Thus it becomes clear that when $\Delta \hat{\phi}=2 \pi$ is fixed, the scaling symmetry of the vacuum (2.1) is broken by the coordinate transformation (2.12), and hence the two metrics are not globally equivalent. An important consequence is that the metric (2.11) has CTCs for $r>r_{c}$ with

$$
\operatorname{coth}\left(\frac{1}{2} r_{c}\right)=\frac{2}{\sqrt{\alpha}} \text {. }
$$

Here $r=r_{c}$ is the velocity of light surface (VLS) for which $g_{\hat{\phi} \hat{\phi}}=0$. We shall call the metric (2.9) the deformed Gödel metric that is asymptotic to the Gödel metric. For general parameters $(a, b)$, there can be two VLS's between which $g_{\phi \phi}>0$. The global structure of the metric (2.9), written in somewhat different parametrization, was analysed in [13].

The emerging of the CTCs in Gödel metrics is a consequence of that $\alpha \leq 1$. If one allows $\alpha>1$, the metrics do not have naked CTCs. However, as we saw earlier that in the framework of Einstein gravity, $\alpha>1$ violates the nullenergy condition. In higher-derivative gravity due to the $\alpha^{\prime}$ correction of string theory, solutions with $\alpha>1$ were constructed in [2]. However, the theory, when treated on its own, involves inevitable ghost modes.

\subsection{Mass and angular momentum}

The general Gödel metric has two Killing vectors,

$\xi_{t}=\frac{1}{\ell^{2}} \frac{\partial}{\partial t}, \quad \xi_{\phi}=\frac{1}{\ell} \frac{\partial}{\partial \phi}$.

Following the Wald formalism [29], which computes the variation of the on-shell Hamiltonian $\delta \mathcal{H}$ associated with a Killing vector with respect to the integration constants of the solutions, we read off the associated conversed quantities by evaluating $\delta \mathcal{H}$ at asymptotic infinity to find

$M=\frac{\sqrt{\alpha} a}{16 \pi} \Delta \phi, \quad J=\frac{\ell \sqrt{\alpha} b}{16 \pi} \Delta \phi$,

where $\Delta \phi$ is given by (2.10). Note that if one chooses a fixed period $\Delta \phi=2 \pi$ instead, rather than the one given by (2.10), the solution will have a naked singularity at $r=r_{0}$, for generic $\alpha$. We have also set the convention $\int d z=1$. For periodic $z$, this means the period is $\Delta z=1$; for real line $z$, this implies that extensive quantities such as $M$ and $J$ are in fact uniform densities over the line $z$.

\subsection{Squashed 3-sphere $S_{\alpha}^{3} \times \mathbb{T}$}

In the Gödel metrics, if we let $\ell^{2}=-\tilde{\ell}^{2}<0$, the metric (2.9) becomes

$\mathrm{d} s^{2}=\tilde{\ell}^{2}\left((\mathrm{~d} z+r \mathrm{~d} \phi)^{2}+\alpha h \mathrm{~d} \phi^{2}+\frac{\mathrm{d} r^{2}}{h}-\mathrm{d} t^{2}\right)$,

where we have swapped the role of $(t, z)$ and have set, without loss of generality, $h=1-r^{2}$. Letting $r=\cos \theta$, we have 
$\mathrm{d} s^{2}=\tilde{\ell}^{2}\left((\mathrm{~d} z+\cos \theta d \phi)^{2}+\alpha \sin ^{2} \theta \mathrm{d} \phi^{2}+\mathrm{d} \theta^{2}-\mathrm{d} t^{2}\right)$.

This metric describes a direct product of time with a squashed 3 -sphere, which we call $S_{\alpha}^{3}$. When the quashing parameter $\alpha=1, S_{\alpha}^{3}$ becomes $S^{3}$, written as a $U(1)$ bundle over $S^{2}$. The regularity of $S_{\alpha}^{3}$ requires that

$\Delta \phi=\frac{2 \pi}{\sqrt{\alpha}}, \quad \Delta z=\frac{4 \pi}{\sqrt{\alpha}}$.

The period $\Delta z$ can be divided by a natural number $n$ without introducing any singularity to the manifold, giving rise to $S_{\alpha}^{3} / \mathbb{Z}_{n}$. (Such a compact Gödel universe was also considered in [26].)

\section{Gödel solutions from Lagrangian formalism}

As mentioned in the introduction, theories in the literature associated with the Gödel universe (2.1) or (2.9) typically involve a matter energy-momentum tensor with unknown Lagrangian origin. A well-known example in four dimensions is the Einstein-Maxwell theory with an axion and a negative cosmological constant [21],

$\mathcal{L}=\sqrt{-g}\left(R-2 \Lambda-\frac{1}{2}(\partial \chi)^{2}-\frac{1}{4} F^{2}\right)$,

where $F=\mathrm{d} A$ is the field strength. For the metric (2.1), the solutions for the matter fields are

$A=\sqrt{2(1-\alpha)} \ell \sin \left(\frac{z}{\sqrt{\alpha}}\right)(\mathrm{d} t+r \mathrm{~d} \phi), \quad \chi=\frac{z}{\sqrt{\alpha}}$,

$\ell^{2}=-\frac{1}{2 \Lambda}$.

In this case, the continuous shifting symmetry along the $z$ direction is broken to a discrete symmetry by the Maxwell potential $A$, which is periodic in $z$. (The axion $\chi$ field does not break this symmetry since only $d \chi$ appears in the theory.) The solution is best described as $\mathrm{G}_{\alpha} \times S^{1}$ rather than $\mathrm{G}_{\alpha} \times$ $\mathbb{R}$. A consequence is that $\mathrm{G}_{\alpha}$ is not a solution to the threedimensional massless sector in the Kaluza-Klein reduction of (3.1) on $z$. In this section, we shall construct more examples of Lagrangians that admit Gödel metrics $\mathrm{G}_{\alpha} \times \mathbb{R}$ as exact solutions.

3.1 Einstein-Maxwell-Axion theory with a topological term

In addition to (3.1), we introduce the additional topological term

$$
\begin{aligned}
\mathcal{L}= & \sqrt{-g}\left(R-2 \Lambda-\frac{1}{4} F^{2}-\frac{1}{2}(\partial \chi)^{2}\right) \\
& +\frac{1}{8} \varepsilon^{\mu \nu \rho \sigma} \chi F_{\mu \nu} F_{\rho \sigma},
\end{aligned}
$$

where $F=\mathrm{d} A$ is the field strength, $\varepsilon^{\mu \nu \rho \sigma}$ is the density of Levi-Civita tensor whose components are $\pm 1,0$. We choose the convention $\varepsilon^{0123}=1$. The axion, Maxwell and Einstein equations of motion are given by

$$
\begin{aligned}
& \partial_{\mu}\left(\sqrt{-g} \partial^{\mu} \chi\right)+\frac{1}{8} \varepsilon^{\mu \nu \rho \sigma} F_{\mu \nu} F_{\rho \sigma}=0, \\
& \partial_{\mu}\left(\sqrt{-g} F^{\mu \nu}-\frac{1}{2} \chi \varepsilon^{\mu \nu \rho \sigma} F_{\rho \sigma}\right)=0, \\
& R_{\mu \nu}-\frac{1}{2} g_{\mu \nu} R+g_{\mu \nu} \Lambda-\frac{1}{2}\left(F_{\mu \rho} F_{\nu}{ }^{\rho}-\frac{1}{4} g_{\mu \nu} F^{2}\right) \\
& \quad-\frac{1}{2}\left(\partial_{\mu} \chi \partial_{\mu} \chi-\frac{1}{2} g_{\mu \nu}(\partial \chi)^{2}\right)=0 .
\end{aligned}
$$

For the general Gödel metric (2.9) with (2.8), we consider the following ansatz for the axion and Maxwell field:

$A=q r \mathrm{~d} \phi, \quad \chi=k z$.

We find that the equations of motion are all satisfied provided that

$k=\frac{1}{\sqrt{\alpha}}, \quad \alpha=1-\frac{q^{2}}{2 \ell^{2}}, \quad \ell^{2}=-\frac{1}{2 \Lambda}$.

The general solution contains three integration constants, $(a, b, q)$. The reality condition requires that $|q|<\sqrt{2} \ell$. It follows that we have $0<\alpha \leq 1$ and $k \geq 1$. We have the original $\alpha=\frac{1}{2}$ Gödel metric corresponding to $q=\ell$. The $\mathrm{AdS}_{3}$ factor arises when $\alpha=1$, corresponding to turning off the Maxwell field. In Sect. 3.3, we consider the case with $\alpha<0$, for which the metric describes $S_{\alpha}^{3} \times \mathbb{T}$.

It is worth pointing out that in four dimensions, the axion $\chi$ is the Hodge dual to a 2 -form potential $B_{(2)}$ with

$G_{(3)}=\mathrm{d} B_{(2)}=* \mathrm{~d} \chi+\frac{1}{2} A \wedge F$.

The Lagrangian (3.3) is equivalent to

$\mathcal{L}=\sqrt{-g}\left(R-2 \Lambda-\frac{1}{4} F^{2}-\frac{1}{12} G_{(3)}^{2}\right)$.

For the Gödel solution we have $B_{(2)}=r d t \wedge d \phi$. The 3-form field strength $G_{(3)}$ is suggestive of string theory, which we shall discuss in Sect. 5.

\subsection{Einstein-Proca-Axion theory}

In this subsection, we replace the previous Maxwell field by a Proca field of mass $\mu$, with the Lagrangian

$$
\mathcal{L}_{2}=\sqrt{-g}\left(R-2 \Lambda-\frac{1}{4} F^{2}-\frac{1}{2} \mu^{2} A^{2}-\frac{1}{2}(\partial \chi)^{2}\right) .
$$

The equations of motion are

$$
\begin{aligned}
& \square \chi=0, \quad \nabla_{\mu} F^{\mu \nu}-\mu^{2} A^{\nu}=0, \\
& R_{\mu \nu}-\frac{1}{2} g_{\mu \nu} R+g_{\mu \nu} \Lambda-\frac{1}{2}\left(F_{\mu \rho} F_{\nu}{ }^{\rho}-\frac{1}{4} g_{\mu \nu} F^{2}\right) \\
& \quad-\frac{1}{2} \mu^{2}\left(A_{\mu} A_{\nu}-\frac{1}{2} g_{\mu \nu} A^{2}\right) \\
& \quad-\frac{1}{2}\left(\partial_{\mu} \chi \partial_{\mu} \chi-\frac{1}{2} g_{\mu \nu}(\partial \chi)^{2}\right)=0 .
\end{aligned}
$$


(There should be no confusion between the Proca mass $\mu$ and the spacetime indices.) The metric ansatz is given by (2.9). We consider the following ansatz for $A$ and $\chi$ :

$A=q(\mathrm{~d} t+r \mathrm{~d} \phi), \quad \chi=z$.

Substituting these into the equations of motion, we find

$\mu=\frac{1}{\ell \sqrt{\alpha}}, \quad \Lambda=\frac{3 q^{2}-2 \ell^{2}}{4 \ell^{2}\left(\ell^{2}-q^{2}\right)}, \quad \alpha=1-\frac{q^{2}}{\ell^{2}}$.

Note that all the constants except for $(a, b)$ appearing in the solution are fixed by the coupling constants of the theory, namely $(\Lambda, \mu)$. It follows that unlike in the earlier EMA theory, the general solution involves only two integration constants. The original $\alpha=\frac{1}{2}$ Gödel metric corresponds to taking $q=\ell / \sqrt{2}$.

\subsection{The embedding of squashed 3-sphere}

In the embedding of the Gödel metric in both EMA and EPA theories discussed above, the cosmological constant $\Lambda$ is negative. When it is positive, the metric $\mathrm{G}_{\alpha} \times \mathbb{R}$ becomes $S_{\alpha}^{3} \times \mathbb{T}$, as in (2.17). For the EMA theory, we have

$$
\begin{aligned}
& A=q \cos \theta \mathrm{d} \phi, \quad \chi=\frac{t}{\sqrt{\alpha}}, \quad \tilde{\ell}^{2}=\frac{1}{2 \Lambda}, \\
& \alpha=1+\frac{q^{2}}{2 \tilde{\ell}^{2}} .
\end{aligned}
$$

For EPA theory, we have

$$
\begin{aligned}
& A=q(\mathrm{~d} z+\cos \theta \mathrm{d} \phi), \quad \chi=t, \quad \mu^{2}=-\frac{1}{\tilde{\ell}^{2} \alpha}, \\
& \Lambda=\frac{3 q^{2}+2 \tilde{\ell}^{2}}{4 \tilde{\ell}^{2}\left(\tilde{\ell}^{2}+q^{2}\right)}, \alpha=1+\frac{q^{2}}{\tilde{\ell}^{2}} .
\end{aligned}
$$

Thus we see that the embedding of the $S_{\alpha}^{3}$ in EPA theory requires a tachyonic vector with $\mu^{2}<0$, while in EMA theory, no exotic matter is required.

\subsection{Generalizing to higher dimensions}

In this section, we generalize the Gödel universe to higher dimensions by considering $\mathrm{G}_{\alpha} \times \mathbb{R}^{n}$, namely

$$
\begin{aligned}
\mathrm{d} s^{2}= & \ell^{2}\left(-(\mathrm{d} t+r \mathrm{~d} \phi)^{2}+\alpha r^{2} f \mathrm{~d} \phi^{2}+\frac{\mathrm{d} r^{2}}{r^{2} f}\right. \\
& \left.+\mathrm{d} z^{i} d z^{i}\right), \quad i=1,2, \ldots, n .
\end{aligned}
$$

The $\alpha=\frac{1}{2}$ solution can be still solved by (1.2), but with $u^{\mu}=\left(\ell^{-2}, 0, \ldots, 0\right)$.

In order for the metrics to be solutions of some Lagrangians, we can replace the axion $\chi$ in the previous subsections by a $(n-1)$-form potential $B_{(n-1)}$ with the field strength
$G_{(n)}=\mathrm{d} B_{(n-1)}$. The Lagrangian (3.3) becomes

$$
\begin{aligned}
\mathcal{L}= & \sqrt{-g}\left[R-2 \Lambda-\frac{1}{4} F^{2}-\frac{1}{2 n !} G_{(n-1)}^{2}\right] \\
& +\frac{1}{8(n-1) !} \varepsilon^{\mu \nu \rho \sigma \alpha_{1} \cdots \alpha_{n-1}} B_{\alpha_{1} \cdots \alpha_{n-1}} F_{\mu \nu} F_{\rho \sigma} .
\end{aligned}
$$

(This should not be confused with dualizing $d \chi$ to the 3form field strength in four dimensions, discussed in the end of Sect. 3.1.) The axion ansatz (3.5) is replaced by

$G_{(n)}=k \mathrm{~d} z_{1} \wedge \cdots \mathrm{d} z_{n}$.

The Lagrangian (3.9) is now replaced by

$\mathcal{L}_{2}=\sqrt{-g}\left(R-2 \Lambda-\frac{1}{4} F^{2}-\frac{1}{2} \mu^{2} A^{2}-\frac{1}{2 n !} G_{(n)}^{2}\right)$.

The corresponding ansatz for $G_{(n)}$ is given by (3.17) with $k=1$.

\subsection{Effective three-dimensional theories}

The non-trivial part of the Gödel universe is the threedimensional metric $\mathrm{G}_{\alpha}$. We can perform dimensional reduction on the coordinate $z$ associated with the trivial $\mathbb{R}$ direction. The EMA theory becomes

$$
\mathcal{L}=\sqrt{-g}\left(R-2 \Lambda_{\text {eff }}-\frac{1}{4} F^{2}\right)+\frac{1}{4} \lambda_{\text {eff }} \varepsilon^{\mu \nu \rho} A_{\mu} F_{v \rho}
$$

In this case, the three-dimensional $\mathrm{G}_{\alpha}$ metric is supported by

$$
\begin{aligned}
& A=q r \mathrm{~d} \phi, \quad \alpha=1-\frac{q^{2}}{2 \ell^{2}}, \quad \Lambda_{\mathrm{eff}}=\frac{q^{2}-\ell^{2}}{2 \ell^{2}\left(2 \ell^{2}-q^{2}\right)}, \\
& \lambda_{\mathrm{eff}}^{2}=\frac{2}{2 \ell^{2}-q^{2}} .
\end{aligned}
$$

The $\mathrm{G}_{\alpha}$ metric of this theory was constructed in [13], where the global structure of $\mathrm{G}_{\alpha}$ was discussed. Under the KaluzaKlein reduction, the EPA theory becomes

$\mathcal{L}=\sqrt{-g}\left(R-2 \Lambda_{\text {eff }}-\frac{1}{4} F^{2}-\frac{1}{2} \mu^{2} A^{2}\right)$.

In this case, the $G_{\alpha}$ metric is supported by

$A=q(\mathrm{~d} t+r \mathrm{~d} \phi), \quad \mu=\frac{1}{\ell \sqrt{\alpha}}, \quad \alpha=1-\frac{q^{2}}{\ell^{2}}$,

$\Lambda_{\mathrm{eff}}=\frac{2 q^{2}-\ell^{2}}{4 \ell^{2}\left(\ell^{2}-q^{2}\right)}$.

In both theories, the free parameters of the solutions are $(a, b)$ of the function $f$ (2.8), while $q$ and hence $\alpha$ are fixed by the coupling constants of the theories. In the above dimensional reductions, we have performed further consistent truncations to the subset of fields that are relevant to the $\mathrm{G}_{\alpha}$ metrics. Note that the solution at the beginning of this section involves a $z$-dependent $A$, and hence it cannot be reduced to the threedimensional massless sector. 


\section{Black holes from double Wick rotations}

\subsection{Type I}

As was discussed in [13], the metrics $\mathrm{G}_{\alpha}$ can describe black holes in three dimensions after double Wick rotations,

$t \rightarrow \mathrm{i} t, \quad \phi \rightarrow \mathrm{i} \phi$

The general metric (2.9) now becomes

$\mathrm{d} s^{2}=\ell^{2}\left((\mathrm{~d} t+r \mathrm{~d} \phi)^{2}-\alpha r^{2} \tilde{f} \mathrm{~d} \phi^{2}+\frac{\mathrm{d} r^{2}}{r^{2} \tilde{f}}+\mathrm{d} z^{2}\right)$,

with the function $\tilde{f}$ now given by

$\tilde{f}=1-\frac{a}{r}-\frac{b}{r^{2}}$.

For EMA theory, we find that the matter fields are given by

$A=q r \mathrm{~d} \phi, \quad \chi=\frac{z}{\sqrt{\alpha}}, \quad$ with $\ell^{2}=-\frac{1}{2 \Lambda}$,

$\alpha=1+\frac{q^{2}}{2 \ell^{2}} \geq 1$.

For EPA theory, we have

$$
\begin{gathered}
A=q(\mathrm{~d} \phi+r \mathrm{~d} t), \quad \chi=z, \quad \text { with } \mu=\frac{1}{\ell \sqrt{\alpha}}, \\
\Lambda=\frac{3 q^{2}+2 \ell^{2}}{4 \ell^{2}\left(\ell^{2}+q^{2}\right)}, \\
\alpha=1+\frac{q^{2}}{\ell^{2}} \geq 1 .
\end{gathered}
$$

The thermodynamics of three-dimensional black holes was studied in [13]. Here we would like to derive the first law in our context and notations. A new subtlety emerges in EMA theory, where the parameter $q$ is an integration constant. For simplicity, we shall set $\ell=1$ for the following discussions. We also assume that the coordinate $\phi$ is periodic with $\Delta \phi=2 \pi$. Thus the solution describes a rotating metric. The null-Killing vector on the horizon $r=r_{0}$ with $f\left(r_{0}\right)=0$ is given by

$\xi=\frac{\partial}{\partial t}-\Omega_{+} \frac{\partial}{\partial \phi}, \quad \Omega_{+}=\frac{1}{r_{0}}$.

It is straightforward to verify that the surface gravity and hence the temperature are given by

$\kappa=\frac{\sqrt{\alpha} r_{0}}{4 \pi} \tilde{f}^{\prime}\left(r_{0}\right), \quad T=\frac{\kappa}{2 \pi}$.

The mass and angular momentum can be read off from the Wald formalism, given by

$M=\frac{1}{8} \sqrt{\alpha} a, \quad J=\frac{1}{8} \sqrt{\alpha} b$.

Two situations emerge at this stage. For black holes of the EPA theory or the effective theories in three dimensions, the parameter $q$ and hence $\alpha$ are fixed constants. In these cases, the first law of black hole thermodynamics reads

$\mathrm{d} M=T \mathrm{~d} S+\Omega_{+} \mathrm{d} J$.

In EMA theory, on the other hand, the parameter $q$ is an integration constant, and hence it can be varied and should be involved in the first law. To complete the first law involving the parameter $q$, we first note that the electric charge of the Maxwell field vanishes, namely

$\int * F+\mathrm{d} \chi \wedge A=0$.

(In [13], an electric charge associated with pure gauge transformation of $A$ was introduced. We shall not consider this here.) The linear charge density of the axion field on the other hand is non-vanishing

$Q_{\chi}=\frac{1}{8} \int \mathrm{d} \chi=\frac{1}{8 \sqrt{\alpha}}$.

The corresponding thermodynamical potential can be read off from the 2-form potential $B_{(2)}$, that is, the Hodge dual to the axion, as in (3.7). It is given by

$\Phi_{\chi}=r_{0}$.

We find that the first law reads

$\mathrm{d} M=T \mathrm{~d} S+\Omega_{+} \mathrm{d} J+\Phi_{\chi} \mathrm{d}\left(\alpha Q_{\chi}\right)$.

It is puzzling that an extra factor $\alpha$ is needed for the completion of the first law above.

\subsection{Type II}

In this interpretation, we switch $t$ and $\phi$ in (4.2) and write the metric as

$\mathrm{d} s^{2}=\ell^{2}\left(\left(\mathrm{~d} \phi+\left(r-r_{0}\right) \mathrm{d} t\right)^{2}-\alpha r^{2} \tilde{f} \mathrm{~d} t^{2}+\frac{\mathrm{d} r^{2}}{r^{2} \tilde{f}}+\mathrm{d} z^{2}\right)$.

Note that we also made a coordinate transformation so that the null-Killing vector at the degenerate surface $r=r_{0}$ with $\tilde{f}\left(r_{0}\right)=0$ is $\xi=\partial_{t}$. In other words, the metric is nonrotating on the horizon. The temperature is given by

$T=\frac{\sqrt{\alpha} r_{0}^{2} \tilde{f}^{\prime}\left(r_{0}\right)}{4 \pi}$.

The solution has no CTCs since $g_{\phi \phi}=\ell^{2}$ and furthermore $g_{t t}>0$ for $r>r_{0}$, and hence $t$ is globally defined outside the horizon. Note that in this case, the entropy is a constant since the radius of the $\phi$ circle is constant. We can also show, using the Wald formalism, that the mass and angular momentum both vanish. The solution can be viewed as a thermalized 
vacuum. In EMA theory, $q$ is an integration constant, which leads to non-zero electric charge and potential, given by

$$
\begin{aligned}
Q_{A} & =\frac{1}{16 \pi} \int * F+\mathrm{d} \chi \wedge A=\frac{q}{16 \pi \sqrt{\alpha}} \int \mathrm{d} \phi \mathrm{d} z \\
& =\frac{q}{16 \pi \sqrt{\alpha}} \Delta \phi, \quad \Phi_{A}=q r_{0} .
\end{aligned}
$$

The axion charge and its thermodynamical potential are given by (4.11) and (4.12). This leads to the first law of black hole "thermodynamics",

$\Phi_{A} \mathrm{~d} Q_{A}+\Phi_{\chi} \mathrm{d} Q_{\chi}=0$

provided that $\Delta \phi=\pi$.

\section{Embedding in string theory}

\subsection{Freedman-Schwarz model}

In Sect. 3 we constructed some ad hoc theories that admit Gödel metrics as exact solutions. The Maxwell and axion fields are common occurrences in supergravities, indicating that there may exist an exact embedding of the Gödel universe in supergravity and hence in string theory. In this section, we consider Freedman-Schwarz $S U(2) \times S U(2)$ gauged supergravity whose bosonic sector consists of the metric, a dilaton $\varphi$, an axion and two $S U(2)$ Yang-Mills fields. After truncating to the $U(1)^{2}$ subsector, the corresponding Lagrangian is

$$
\begin{aligned}
\mathcal{L}= & \sqrt{-g}\left(R-\frac{1}{2}(\partial \varphi)^{2}-\frac{1}{2} e^{2 \varphi}(\partial \chi)^{2}\right. \\
& \left.+2\left(g_{1}^{2}+g_{2}^{2}\right) e^{\varphi}-\frac{1}{4} e^{-\varphi}\left(F_{1}^{2}+F_{2}^{2}\right)\right) \\
& +\frac{1}{8} \varepsilon^{\mu \nu \rho \sigma} \chi\left(F_{1 \mu \nu} F_{1 \rho \sigma}+F_{2 \mu \nu} F_{2 \rho \sigma}\right),
\end{aligned}
$$

where $\left(g_{1}, g_{2}\right)$ are the gauge coupling constants of the two $S U$ (2) Yang-Mills fields. The theory admits the general deformed Gödel metric (2.9) with the matter fields given by

$A_{i}=q_{i} r \mathrm{~d} \phi, \quad \chi=\frac{z}{\sqrt{\alpha}}, \quad \varphi=0$,

with the parameters

$\ell^{2}=\frac{1}{2\left(g_{1}^{2}+g_{2}^{2}\right)}, \quad \alpha=1-\left(g_{1}^{2}+g_{2}^{2}\right)\left(q_{1}^{2}+q_{2}^{2}\right)$.

\subsection{Gödel universe from string theory}

The Freedman-Schwarz model can be obtained from the Kaluza-Klein reduction on $S^{3} \times S^{3}$ [27,28]. The relevant part of the effective Lagrangian of strings in ten dimensions is

$\mathcal{L}_{10}=\sqrt{-g}\left(R-\frac{1}{2}(\partial \Phi)^{2}-\frac{1}{12} e^{-\Phi} F_{(3)}^{2}\right)$, where $F_{(3)}$ can be either NS-NS or R-R fields. Following the reduction ansatz given in [28], we find that the tendimensional solution is given by $\Phi=0$, together with

$$
\begin{aligned}
\mathrm{d} s_{10}^{2}= & \frac{1}{g_{1}^{2}+g_{2}^{2}}\left(-(\mathrm{d} t+r \mathrm{~d} \phi)^{2}\right. \\
& \left.+\alpha r^{2} f \mathrm{~d} \phi^{2}+\frac{\mathrm{d} r^{2}}{r^{2} f}+\mathrm{d} z^{2}\right) \\
& +\frac{1}{g_{1}^{2}}\left(\left(\mathrm{~d} \psi_{1}+\cos \theta_{1} \mathrm{~d} \phi_{1}+g_{1} q_{1} r \mathrm{~d} \phi\right)^{2}\right. \\
& \left.+\mathrm{d} \theta_{1}^{2}+\sin ^{2} \theta_{1} \mathrm{~d} \phi_{1}^{2}\right) \\
& +\frac{1}{g_{2}^{2}}\left(\left(\mathrm{~d} \psi_{2}+\cos \theta_{2} \mathrm{~d} \phi_{2}\right.\right. \\
& \left.\left.+g_{2} q_{2} r \mathrm{~d} \phi\right)^{2}+\mathrm{d} \theta_{2}^{2}+\sin ^{2} \theta_{2} \mathrm{~d} \phi_{2}^{2}\right), \\
F_{(3)}= & \frac{1}{g_{1}^{2}+g_{2}^{2}} \mathrm{~d} t \wedge \mathrm{d} r \wedge \mathrm{d} \phi \\
& -\frac{\sin \theta_{1}}{g_{1}^{2}} \mathrm{~d} \theta_{1} \wedge \mathrm{d} \phi_{1} \wedge\left(\mathrm{d} \psi_{1}+\cos \theta_{1} \mathrm{~d} \phi_{1}+g_{1} q_{1} r \mathrm{~d} \phi\right) \\
& -\frac{\sin \theta_{2}}{g_{2}^{2}} \mathrm{~d} \theta_{2} \wedge \mathrm{d} \phi_{2} \wedge\left(\mathrm{d} \psi_{2}+\cos \theta_{2} \mathrm{~d} \phi_{2}+g_{2} q_{2} r \mathrm{~d} \phi\right) \\
& -\frac{q_{1}}{g_{1}} \mathrm{~d} r \wedge \mathrm{d} \phi \wedge\left(\mathrm{d} \psi_{1}+\cos \theta_{1} \mathrm{~d} \phi_{1}\right) \\
& -\frac{q_{2}}{g_{2}} \mathrm{~d} r \wedge \mathrm{d} \phi \wedge\left(\mathrm{d} \psi_{2}+\cos \theta_{2} \mathrm{~d} \phi_{2}\right) .
\end{aligned}
$$

Here $\alpha$ is again given by (5.3). The solution involves both electric string and magnetic fivebrane/fivebrane charges, given by

electric : $Q_{1} \sim \int * F_{(3)} \sim \frac{1}{\sqrt{\alpha} g_{1}^{3} g_{2}^{3}}$,

magnetic : $Q_{5 / 5} \sim \int F_{(3)} \sim \frac{1}{g_{1}^{2}}+\frac{1}{g_{2}^{2}}$.

These can be either all NS-NS charges or R-R charges, and the latter corresponds to the D1/D5/D5 configuration. When $q_{1}=0=q_{2}$, the metric becomes $\operatorname{AdS}_{3} \times S^{3} \times S^{3} \times \mathbb{R}$, which is the decoupling limit of the string/fivebrane/fivebrane configuration [30]. The rotations associated with parameters $\left(q_{1}, q_{2}\right)$ turn the $\mathrm{AdS}_{3}$ into the $\mathrm{G}_{\alpha}$. We thus expect that there should be a rotating string/fivebrane/fivebrane configuration whose decoupling limit gives rise to our ten-dimensional solution (5.6). If we set either $g_{1}=0$ or $g_{2}=0$, but not both, the associated $S^{3}$ is flattened to become $\mathbb{R}^{3}$. The metric configuration becomes $G_{\alpha} \times S^{3} \times \mathbb{R}^{4}$. The heterotic string solution of $G_{\alpha} \times S^{3} \times K_{3}$ was first constructed in [11].

To study the global structure, we first denote $r_{0}$ as the largest root of $f(r)$. Shifting the coordinates,

$t \rightarrow t-r_{0} \phi, \quad \psi_{i} \rightarrow \psi_{i}-g_{i} q_{i} r_{0} \phi$ 
we find that the metric is singular at $r=r_{0}$, where the degenerate Killing vector is purely spatial $\xi=\partial_{\phi}$. The absence of a conical singularity requires that

$\Delta \phi=\frac{4 \pi}{\sqrt{\alpha} r_{0}^{2} f^{\prime}\left(r_{0}\right)}$.

Thus, in this system, there are three periodic coordinates $\phi$, and $\left(\psi_{1}, \psi_{2}\right)$, with $\Delta \psi_{1}=4 \pi=\Delta \psi_{2}$. The coordinate $t$ on the other hand is not required to be periodic. The analysis of CTCs in ten dimensions becomes more subtle. Note that we have

$g_{\phi \phi}=\frac{\alpha r_{0}^{2} f^{\prime}\left(r_{0}\right)}{g_{1}^{2}+g_{2}^{2}}\left(r-r_{0}\right) \geq 0$,

for the region $r \geq r_{0}$; however, naked CTCs still exist. One way to see this is to consider the general periodic Killing vector,

$\xi=\beta \frac{\partial}{\partial \phi}+\gamma_{1} \frac{\partial}{\partial \psi_{1}}+\gamma_{2} \frac{\partial}{\partial \psi_{2}}$.

The absence of naked CTCs requires that $\xi^{2} \geq 0$ for all real $\left(\beta, \gamma_{1}, \gamma_{2}\right)$ in the $r \geq r_{0}$ region. This can be easily established not true. Negative modes arise for large enough $r$.

A simpler way to see that naked CTCs exist is as follows. Let $r=r_{*}$ such that $r_{*}^{2}\left(\alpha f\left(r^{*}\right)-1\right)<0$, which is always achievable since $0<\alpha<1$. Now making a coordinate shift $\psi_{i} \rightarrow \psi_{i}-g_{i} q_{i} r_{*} \phi$, then we have $g_{\phi \phi}=r_{*}^{2}\left(\alpha f\left(r^{*}\right)-1\right)<$ 0 .

\section{$5.3 \mathrm{AdS}_{3} \times S^{3}$ bundle over $S_{\alpha}^{3}$}

We now consider the case with negative $g_{1}^{2}=-\hat{g}_{1}^{2}$ and $\hat{g}_{1}^{2}-$ $g_{2}^{2}>0$. Performing some appropriate analytical continuation of the coordinates on the solution (5.6) and then dropping the hat symbol, we have

$$
\begin{aligned}
\mathrm{d} s_{10}^{2}= & \frac{1}{g_{1}^{2}-g_{2}^{2}}\left((\mathrm{~d} \psi+\cos \theta \mathrm{d} \phi)^{2}\right. \\
& \left.+\alpha \sin ^{2} \theta \mathrm{d} \phi^{2}+\mathrm{d} \theta^{2}+\mathrm{d} z^{2}\right) \\
& +\frac{1}{g_{1}^{2}}\left(\left(\mathrm{~d} \psi_{1}+\rho \mathrm{d} t+g_{1} q_{1} \cos \theta \mathrm{d} \phi\right)^{2}\right. \\
& \left.+\frac{\mathrm{d} \rho^{2}}{\rho^{2}+1}-\left(\rho^{2}+1\right) \mathrm{d} t^{2}\right) \\
& +\frac{1}{g_{2}^{2}}\left(\left(\mathrm{~d} \psi_{2}+\cos \theta_{2} \mathrm{~d} \phi_{2}+g_{2} q_{2} \cos \theta \mathrm{d} \phi\right)^{2}\right. \\
& \left.+\mathrm{d} \theta_{2}^{2}+\sin ^{2} \theta_{2} \mathrm{~d} \phi_{2}^{2}\right) \\
F_{(3)}= & -\frac{1}{g_{1}^{2}-g_{2}^{2}} \mathrm{~d} \psi \wedge \mathrm{d} \cos \theta \wedge \mathrm{d} \phi \\
& +\frac{1}{g_{1}^{2}} \mathrm{~d} \rho \wedge \mathrm{d} t \wedge\left(\mathrm{d} \psi_{1}+\rho \mathrm{d} t+g_{1} q_{1} \cos \theta \mathrm{d} \phi\right)
\end{aligned}
$$

$$
\begin{aligned}
& -\frac{\sin \theta_{2}}{g_{2}^{2}} \mathrm{~d} \theta_{2} \wedge \\
& \mathrm{d} \phi_{2} \wedge\left(\mathrm{d} \psi_{2}+\cos \theta_{2} \mathrm{~d} \phi_{2}+g_{2} q_{2} \cos \theta \mathrm{d} \phi\right) \\
& -\frac{q_{1}}{g_{1}} \mathrm{~d} \cos \theta \wedge \mathrm{d} \phi \wedge\left(\mathrm{d} \psi_{1}+\rho \mathrm{d} t\right) \\
& -\frac{q_{2}}{g_{2}} \mathrm{~d} \cos \theta \wedge \mathrm{d} \phi \wedge\left(\mathrm{d} \psi_{2}+\cos \theta_{2} \mathrm{~d} \phi_{2}\right),
\end{aligned}
$$

where $\alpha=1+\left(g_{1}^{2}-g_{2}^{2}\right)\left(q_{1}^{2}+q_{2}^{2}\right)$. The solution describes a direct production of a line of coordinate $z$ and a ninedimensional metric of an $\operatorname{AdS}_{3} \times S^{3}$ bundle over the squashed 3 -sphere $S_{\alpha}^{3}$. Again the configuration involves both electric string and magnetic fivebrane/fivebrane charges

electric : $Q_{1} \sim \frac{g_{1}}{\sqrt{\alpha} g_{2}^{2}\left(g_{1}^{2}-g_{2}^{2}\right)^{2}}$,

magnetic : $Q_{5 / 5} \sim \frac{1}{\alpha\left(g_{1}^{2}-g_{2}^{2}\right)}+\frac{1}{g_{2}^{2}}$.

Note that $\Delta \psi \sim \Delta \phi \sim 1 / \sqrt{\alpha}$. When $q_{1}=0=q_{2}$, the metric is again $\operatorname{AdS}_{3} \times S^{3} \times S^{3}$, equivalent to the previous static case. For non-vanishing $q_{i}$ 's, the brane configuration is not clear and it deserves further study. Interestingly, the limit of $g_{1}=g_{2}$ leads to the well-known $\mathrm{AdS}_{3} \times S^{3} \times \mathbb{R}^{4}$ vacuum of string theory, and the limit $g_{2}=0$ gives rise to $\mathrm{AdS}_{3} \times S_{\alpha}^{3} \times \mathbb{R}^{4}$.

\section{Conclusions}

Four-dimensional Gödel metrics of $\mathrm{G}_{\alpha} \times \mathbb{R}$ are perhaps the simplest solutions that exhibit naked CTCs with no globally spatial-like Cauchy horizon. In this paper, we showed that the Gödel metrics could arise as exact solutions in Lagrangian formalism. We constructed EMA and EPA theories that admit Gödel solutions. We also showed that Gödel universe could emerge from Freedman-Schwarz $S U(2) \times S U(2)$ gauge supergravity. This allows us to give exact embeddings of the Gödel metrics in string theories. (It seems very closely related to a T-dual version of the M-theory Gödel solution [31,32].) The ten-dimensional solution describes a direct product of a line and an $S^{3} \times S^{3}$ bundle over $\mathrm{G}_{\alpha}$. Classically, we find that naked CTCs persist in higher dimensions. (In [11], string quantization was performed on $\mathrm{G}_{\alpha} \times S^{3} \times K_{3}$ and it was demonstrated that CTCs can resolved by the quantum effects.) For some appropriate choice of parameters, the nine-dimensional metric can describe an $\mathrm{AdS}_{3} \times S^{3}$ bundle over a squashed 3-sphere $S_{\alpha}^{3}$, in which case there is no CTC. In the suitable limit, the solution becomes the supersymmetric $\mathrm{AdS}_{3} \times S^{3} \times \mathbb{R}^{4}$ vacuum.

The scaling symmetry of the metric (2.1) resembles that of the anti-de Sitter spacetimes. This is suggestive of there possibly existing a boundary field theory at the $r \rightarrow \infty$ boundary of the Gödel universe. The exact embedding of the 
Gödel metrics in string theory, as the decoupling limit of the rotating D1/D5/D5 intersection, provides a tool of investigating the boundary field theory in the context of string theory.

Acknowledgements S.-L.L. and H.W. are supported in part by NSFC under Grant Nos. 11575022 and 11175016 . X.-H.F. and H.L. are supported in part by NSFC Grant Nos. 11175269, 11475024 and 11235003.

Open Access This article is distributed under the terms of the Creative Commons Attribution 4.0 International License (http://creativecomm ons.org/licenses/by/4.0/), which permits unrestricted use, distribution, and reproduction in any medium, provided you give appropriate credit to the original author(s) and the source, provide a link to the Creative Commons license, and indicate if changes were made.

Funded by SCOAP ${ }^{3}$.

\section{References}

1. K. Gödel, An example of a new type of cosmological solutions of Einstein's field equations of graviation. Rev. Mod. Phys. 21, 447 (1949). doi:10.1103/RevModPhys.21.447

2. J.D. Barrow, M.P. Dabrowski, Gödel universes in string theory. Phys. Rev. D 58, 103502 (1998). doi:10.1103/PhysRevD.58. 103502. arXiv:gr-qc/9803048

3. J.P. Gauntlett, J.B. Gutowski, C.M. Hull, S. Pakis, H.S. Reall, All supersymmetric solutions of minimal supergravity in fivedimensions. Class. Quant. Grav. 20, 4587 (2003). doi:10.1088/ 0264-9381/20/21/005. arXiv:hep-th/0209114

4. C.A.R. Herdeiro, Spinning deformations of the D1-D5 system and a geometric resolution of closed timelike curves. Nucl. Phys. B 665, 189 (2003). doi:10.1016/S0550-3213(03)00484-X. arXiv:hep-th/0212002

5. E.K. Boyda, S. Ganguli, P. Horava, U. Varadarajan, Holographic protection of chronology in universes of the Gödel type. Phys. Rev. D 67, 106003 (2003). doi:10.1103/PhysRevD.67.106003. arXiv:hep-th/0212087

6. T. Harmark, T. Takayanagi, Supersymmetric Gödel universes in string theory. Nucl. Phys. B 662, 3 (2003). doi:10.1016/ S0550-3213(03)00349-3. arXiv:hep-th/0301206

7. H. Takayanagi, Boundary states for supertubes in flat spacetime and Gödel universe. JHEP 0312, 011 (2003). doi:10.1088/ 1126-6708/2003/12/011. arXiv:hep-th/0309135

8. D. Brecher, P.A. DeBoer, D.C. Page, M. Rozali, Closed timelike curves and holography in compact plane waves. JHEP 0310(031), 2003 (2003). doi:10.1088/1126-6708/2003/10/031. arXiv:hep-th/0306190

9. D. Brace, C.A.R. Herdeiro, S. Hirano, Classical and quantum strings in compactified pp waves and Gödel type universes. Phys. Rev. D 69, 066010 (2004). doi:10.1103/PhysRevD.69.066010. arXiv:hep-th/0307265

10. E.G. Gimon, A. Hashimoto, Black holes in Gödel universes and pp waves. Phys. Rev. Lett. 91, 021601 (2003). doi:10.1103/ PhysRevLett.91.021601. arXiv:hep-th/0304181

11. D. Israel, Quantization of heterotic strings in a Gödel/anti-de Sitter space-time and chronology protection. JHEP 0401, 042 (2004). doi:10.1088/1126-6708/2004/01/042. arXiv:hep-th/0310158

12. M. Cvetič, G.W. Gibbons, H. Lü, C.N. Pope, Rotating black holes in gauged supergravities: Thermodynamics, supersymmetric limits, topological solitons and time machines. arXiv:hep-th/0504080

13. M. Banados, G. Barnich, G. Compere, A. Gomberoff, Three dimensional origin of Gödel spacetimes and black holes. Phys. Rev. D 73, 044006 (2006). doi:10.1103/PhysRevD.73.044006. arXiv:hep-th/0512105
14. S.Q. Wu, General non-extremal rotating charged Gödel black holes in minimal five-dimensional gauged supergravity. Phys. Rev. Lett. 100, 121301 (2008). doi:10.1103/PhysRevLett.100.121301. arXiv:0709.1749 [hep-th]

15. G. Barnich, G. Compere, Conserved charges and thermodynamics of the spinning Gödel black hole. Phys. Rev. Lett. 95, 031302 (2005). doi:10.1103/PhysRevLett.95.031302. arXiv:hep-th/0501102

16. J.J. Peng, S.Q. Wu, Extremal Kerr black hole/CFT correspondence in the five dimensional Gödel universe. Phys. Lett. B 673 , 216 (2009). doi:10.1016/j.physletb.2009.02.020. arXiv:0901.0311 [hep-th]

17. S.Q. Wu, J.J. Peng, Thermodynamics and Hawking radiation of five-dimensional rotating charged Gödel black holes. Phys. Rev. D 83, 044028 (2011). doi:10.1103/PhysRevD.83.044028. arXiv: 1101.5474 [hep-th]

18. A. Banerjee, S. Banerji, Stationary distributions of dust and electromagnetic fields in general relativity. J. Phys. A 1, 188 (1968)

19. F. Bampi, C. Zordan, A note on Gödel's metric. Gen. Relat. Grav. 9, 393 (1978)

20. A.K. Raychaudhuri, S.N.G. Thakurta, Homogeneous space-times of the Gödel type. Phys. Rev. D 22, 802 (1980). doi:10.1103/ PhysRevD.22.802

21. M.J. Reboucas, J. Tiomno, On the homogeneity of Riemannian space-times of Gödel type. Phys. Rev. D 28, 1251 (1983). doi:10. 1103/PhysRevD.28.1251

22. M.J. Reboucas, J.E. Aman, A.F.F. Teixeira, A note on Gödel type space-times. J. Math. Phys. 27, 1370 (1986). doi:10.1063/1.527093

23. M.J. Reboucas, J. Santos, Gödel-type universes in $f(R)$ gravity. Phys. Rev. D 80, 063009 (2009). doi:10.1103/PhysRevD.80. 063009. arXiv:0906.5354 [astro-ph.CO]

24. J.A. Agudelo, J.R. Nascimento, A.Y. Petrov, P.J. Porfírio, A.F. Santos, Gödel and Gödel-type universes in Brans-Dicke theory. Phys. Lett. B 762, 96 (2016). arXiv: 1603.07582 [hep-th]

25. D.Z. Freedman, J.H. Schwarz, $N=4$ supergravity theory with local $S U(2) \times S U(2)$ invariance. Nucl. Phys. B 137, 333 (1978). doi:10.1016/0550-3213(78)90526-6

26. D. Israel, C. Kounnas, D. Orlando, P.M. Petropoulos, Electric/magnetic deformations of $S^{3}$ and $\mathrm{AdS}_{3}$, and geometric cosets. Fortsch. Phys. 53, 73 (2005). doi:10.1002/prop.200410190. arXiv:hep-th/0405213

27. A.H. Chamseddine, M.S. Volkov, Non-Abelian solitons in $N=4$ gauged supergravity and leading order string theory. Phys. Rev. D 57, 6242 (1998). doi:10.1103/PhysRevD.57.6242. arXiv:hep-th/9711181

28. M. Cvetič, H. Lü, C.N. Pope, Four-dimensional $N=4, S O(4)$ gauged supergravity from $D=11$. Nucl. Phys. B 574, 761 (2000). doi:10.1016/S0550-3213(99)00828-7. arXiv:hep-th/9910252

29. R.M. Wald, Black hole entropy is the Noether charge. Phys. Rev. D 48(8), R3427 (1993). doi:10.1103/PhysRevD.48.R3427. arXiv: gr-qc/9307038

30. P.M. Cowdall, P.K. Townsend, Gauged supergravity vacua from intersecting branes. Phys. Lett. B 429, 281 (1998). Erratum: [Phys. Lett. B 434, 458 (1998)] doi:10.1016/S0370-2693(98)00768-0, 10. 1016/S0370-2693(98)00445-6. arXiv: hep-th/9801165

31. J. Raeymaekers, W. Van Herck, B. Vercnocke, T. Wyder, 5D fuzzball geometries and 4D polar states. JHEP 0810, 039 (2008). doi:10.1088/1126-6708/2008/10/039. arXiv:0805.3506 [hep-th]

32. T.S. Levi, J. Raeymaekers, D. Van den Bleeken, W. Van Herck, B. Vercnocke, Gödel space from wrapped M2-branes. JHEP 1001, 082 (2010). doi:10.1007/JHEP01(2010)082. arXiv:0909.4081 [hep-th] 